Physical Sciences | Alfred Msezane

\section{A rigorous model of electron attachment in lanthanide atoms}

\begin{tabular}{|c|c|}
\hline $\begin{array}{l}\text { Understanding the physical } \\
\text { mechanism underlying } \\
\text { negative ion formation in } \\
\text { low-energy electron collisions } \\
\text { with lanthanide atoms is of } \\
\text { fundamental importance in } \\
\text { physics and chemistry. It also } \\
\text { has important implications for } \\
\text { a wide range of applications, } \\
\text { from catalysis to drug delivery } \\
\text { and water purification. } \\
\text { Unfortunately, the complexity } \\
\text { of the interactions among } \\
\text { electrons in lanthanides has for } \\
\text { a long time made it virtually } \\
\text { impossible to reliably predict } \\
\text { the energetics of the electron } \\
\text { binding and the properties of } \\
\text { the resulting negative ions. } \\
\text { Professor Alfred Msezane } \\
\text { and his group at Clark Atlanta } \\
\text { University have developed } \\
\text { a rigorous and powerful } \\
\text { theoretical description of the } \\
\text { electron attachment process in } \\
\text { lanthanide atoms. }\end{array}$ & $\begin{array}{l}\text { anthanides, along with the } \\
\text { actinide atoms, are a group of } \\
\text { atoms that occupy large blocks } \\
\text { near the bottom of the Periodic } \\
\text { Table. They are heavy and complex } \\
\text { atoms, containing between } 57 \text { and } \\
71 \text { electrons orbiting in complex } \\
\text { trajectories around a positive nucleus, } \\
\text { with velocities of about } 1 \% \text { the speed } \\
\text { of light. Lanthanides play important } \\
\text { roles in modern chemistry and exhibit } \\
\text { a number of unique properties, making } \\
\text { them suitable for applications in } \\
\text { catalysis, superconducting devices, } \\
\text { optoelectronics and fluorescent } \\
\text { materials for drug discovery. They are } \\
\text { also used to regulate nuclear reactors. }\end{array}$ \\
\hline
\end{tabular}

\section{Tb Pm Sm Eu Dy} La Ce Pr Nd Gd lanthanides large negatively charged ions, or anions. The prospect of understanding anionic species is of extraordinary access to unprecedented and exotic

chemical processes, which have great technological and societal impact. For instance, heavy negative ions can provide efficient and inexpensive catalysts for water purification in chemical reactions that involve the conversion of water into hydrogen applied to the large-scale processing of water for civil uses in developing countries.

Professor Msezane's research is devoted to developing rigorous and accurate ho physical phenoms to account for hen lanthanide systems, such as actinide atoms and energy electrons. He has formulated a novel mathematical approach - called the Regge pole method - for the prediction of electron attachment to stable negative ions. The method is proving to be a major theoretical breakthrough in unveiling the complex quantum-mechanical interactions at play during the formation of lanthanide anions.

The central physical measure for the electron is called to bind an excess of the atom. This can be defined as the and con perspective, but it can also give peroxide. This can potentially be fullerene molocus) interato with lowheavy many-electron systems, yielding

\section{ELECTRON AFFINITY}

energy released when one electron added to a neutral atom to form reliable values of electron affinities for complex heavy systems like lanthanides, actinides, and fulleren molecules is currently one of the most challenging problems in atomic and molecular physics, both experimentally and theoretically.

Theoretical models of electron affinity determination must consider that a large number of electrons with the atom are interacting among themselves, and with the incoming low-energy electron. This gives rise to two important quantum-mechanical phenomena - the electron-electron correlation and the core polarisation interaction - which play a crucial process. Although sophisticated computational approaches, such as the relativistic configuration interaction method, can be used to estimate electron affinities, their use is often cumbersome, demanding and plagued by uncertainty, because of the large number of electron configurations which must be included in the calculations in the case of lanthanides. Also, the interpretation of the calculated results can be plagued by mbiguities.

\section{THE ELECTRON}

\section{ATTACHMENT MECHANIS} As a lowe g electon (ncidont) aprom, it initially travels along a linear path, similar to a bullet approaching path, sim tow a buls in appricing of the atom, the complex interaction between the incident electron and the electrons belonging to the atom causes the electron to deviate (or 'scatter') from its original path. The magnitude of this deviation is linked to a quantum-mechanical property of the incident electron (its complex angular momentum) and to its velocity which is related to ts kinetic energy. In some cases, the effect of the scattering can be suticient for the electron to become trapped. In this situation, the (lectron effectively occupies a new stable quantum-mechanical state with the atom, and the sy

\section{Energy $\angle \quad$ Neutral atom Sodium $\quad$ Positive ion}

(5)

- 83 .

- 20 ,

Electron Neutral atom
Chlorine

Negative io
Chlorine

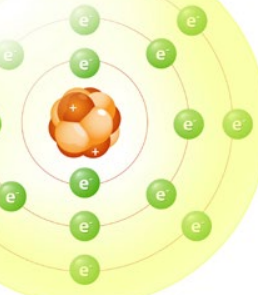

(a)

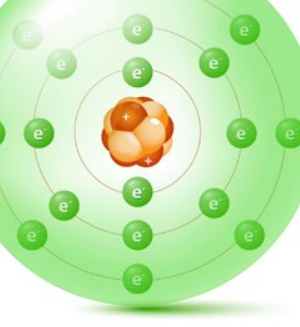

The ability of an atom to bind an excess electron is called the electron affinity of the atom

Msezane has formulated a novel mathematical approach - called the Regge pole method - for low-energy electron collision with heavy manyelectron systems.

The Regge pole method, developed by the Regge pole method is its unique Msezane, is capable of accounting for the Re a lity to identiry the essential states complexity a provides quatitive the collision, and to determing estimates of electron affinities. Crucially, unambiguously the binding energy Msezane's approach does not require of its ground state, from which the input from either experiments or other

theory; it can therefore be used to study electron attachment in for which measured values are not

of experimental data.

It is important to realise that the incident electron may not always occupy the ground state of the newly formed anion. Metastable and excited states can in fact also play a role in the anion formation, and they can substantially complicate measurements and theoretical calculations. One of the strengths of

\section{CROSS SECTIONS}

The central quantity determining the during anion formation in low-energy electron scattering experiments is the elastic total cross section. It can be interpreted as a cross sectional electron binding energy, which is the minimum energy required to form a effectiveness of scattering interactions within whe vicinity of the neutral atom interactions cuantum-mechanical lectron to experiene incoming The second important quantity is the 

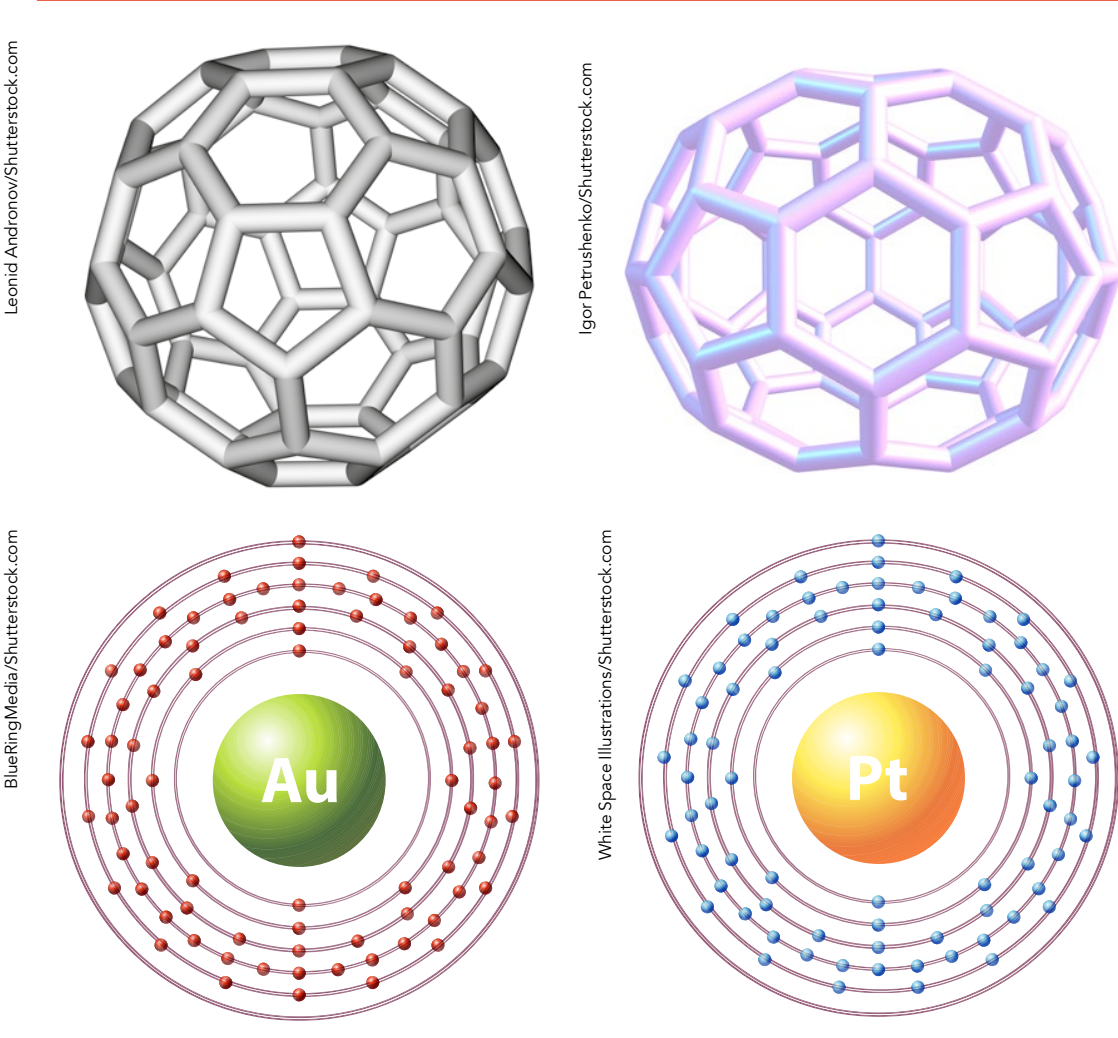

It provides a robust and reliable method of calculating unambiguously electron affinities for the first time.

stable negative ion. The Regge pole method provides the unprecedented ability to calculate anionic binding energies of complex systems from the characteristic sharp peaks appearing in the calculated elastic total cross sections. The ground state determine the electron affinities of the systems.

The robustness and reliability of Msezane's method has been benchmarked by comparison to measured electron affinities of Pt atoms, and the highly radioactive At atom. "This theoretical feat", explains Msezane, "has never been accomplished before. This innovative approach to extracting reliable electron affinities of complex heavy systems from the ground state electron elastic total cross section calculations represents an unprecedented theoretical and reliable method of calculating the first time."

AT WOR

Regge poles, singularities of the resonances and in the physical she to bound states. This explains the great success of the Regge pole method in calculating reliable ground and excited states binding energies of negative ions formed in low-energy electron collisions with complex heavy systems, such as the lanthanides.

In addition to providing robust calculations of electron affinities, the Regge pole method offers a reconciling and rationalising results sophisticated theoretical methods, such as configuration interaction

\section{THE REGGE POLE} of the complex plane they correspond obtained from experiments and from of the ambiguities that make these data difficult to interpret. "Many and sophisticated theoretical calculations", says Msezane "have considered the anionic binding energies of the stable, metastable and/or excited negative ion formation to correspond to the electron affinities of the considered lanthanide and actinide atoms. This is contrary to the usual meaning of the electron affinities found in standard measurements of complex systems, such as Au, Pt, At and fullerene molecules. "Indeed, there are cases in which conventional theoretical methods or experimental measurements fail to provide a correct interpretation of the electron affinities, and they can yield binding energies mecharical states of the alow which are not the ground state.

Professor Msezane and his collaborato Dr Zineb Felfli have demonstrated this to be the case in an elegant study of the theoretically and experimentally determined electron affinities of the 'lighter' lanthanides: $\mathrm{Eu}, \mathrm{Tb}, \mathrm{Tm}$, Gd, (and also the non-lanthanide $\mathrm{Nb}$ ). Using the Regge pole approach, they have shown that all previously reported values for the electron affinities of these atoms correspond to binding energies for their anions in excited states, and they cannot therefore be rigorously interpreted as electron affinities. A new list of 'physical' and sing the Regge pole method, is also using the Regge pole method, is also the atoms examined. The Regge pole approach has also been applied to the 'heavy' lanthanides: Ho, Er, Tm, Yb, and $\mathrm{Lu}$ (as well as to $\mathrm{Hf}$ ). Again, this has shown that the currently available measured and calculated electron affinities are riddled with substantial ambiguities arising from the potentia misidentification of the binding energies of the anions and a new set of electron affinities has been proposed.

These findings highlight the extraordinary potential of the Regge pole method as a revolutionary, rigorous, and robust approach to the calculation many-electron atoms ond momplex
Research Objectives

Professor Alfred Msezane has developed a new robust

\section{Detail}

\section{Address}

Department of Physics \& CTSPS, Clark Atlanta University A23ame P. Brawley Drve SW

Bio Director of CTSPS at Clark Atlanta University is a Fellow of Royal Society of Chemistry, American Physica Society, Institute of Physics and AAAS. He holds B.Sc (Hons), MSc and PhD degrees in physics.

\section{Funding}

Research was supported by the US DOE, Division of Chemical Sciences, Geosciences and Biosciences, Office of Basic Energy Sciences, Office of Energy Research. The computing facilities of the National Energy Research Scientic Computing Center acknowledge support from the US DOE.

Collaborators method for calculating the electron attachment process

Alfred Msezane, Professor of Physics and Founding

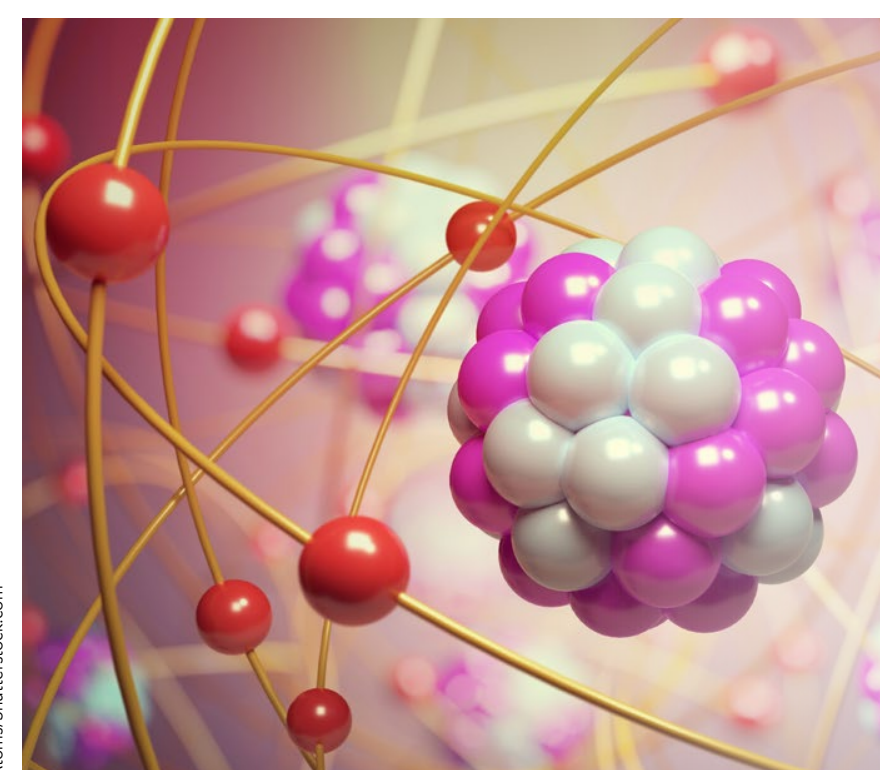

\section{References}

Felfli, Z Msezane, AZ (2018). Conundrum in Measured Electron Affinities of Complex Heavy Atoms. Journal of http://dx.doi.org/10.26713\%2Fjamcnp.v5i2.1093

Felfli, Z Msezane, AZ (2019). Low-energy electron scattering from atomic $\mathrm{Th}, \mathrm{Pa}, \mathrm{U}, \mathrm{Np}$ and Pu: Negative ion formation. Applied Physics Research, 11, 52. DOI:

Felfli, Z Msezane, AZ (2020). Low-Energy Electron Elastic Total Cross Sections for Ho, Er, Tm, Yb, Lu, and Hf Atoms. Atoms, 8, 17. https://doi.org/10.3390/atoms8020017

Msezane, AZ (2021). Alfred Msezane. [online] Wikipedia. [Accessed 25 July 2021]

\section{Personal Response}

\section{What are the key features of the Regge pole}

method that you have developed, and what future applications are you envisioning that will benefit
most from your approach?

II Regge poles are generalised bound states; they section and core-polarization interaction are major in low-energy electron scattering. The robust potential used incorporates the latter effect, whose cross sections. The resonance positions determine metastable, excited and ground states anionic binding electron affinities. We continue to investigate electron affinities for complex systems, required for novel nanocatalysts for air pollution elimination, clean water
and green energy. Atomic, Molecular, Condensate \& Nano Physics, 5(2), 1-8. most from your approach? rigorously define resonances. Electron-electron correlation, fully embedded in the elastic total cross parameters are tuned for resonances in the elastic total 